\title{
Vulnerabilidade social, individual e programática em idosos da comunidade: dados do estudo FIBRA, Campinas, SP, Brasil
}

\author{
Social, individual and programmatic vulnerability \\ among the elderly in the community: data from the FIBRA Study \\ conducted in Campinas, São Paulo, Brazil
}

Natália Oliveira Rodrigues ${ }^{1}$

Anita Liberalesso Neri ${ }^{1}$

${ }^{1}$ Programa de pósGrauação em

Gerontolologia, Faculdade de Ciências Médicas,

Universidade Estadual de Campinas. Av. Tessália

Vieira de Camargo 126 Cidade Universitária

“Zeferino Vaz", Barão

Geraldo. 13083-887

Campinas SP

anitalbn@uol.com.br
Abstract Sociocultural and economic conditions interact with biological processes throughout the course of life determining vulnerability or resilience in old age. The scope of this study was to investigate relationships between social vulnerability (gender, age and income); individual vulnerability (comorbidities, signs and symptoms, functional ability, perceived social support and perceived health), and programmatic vulnerability (indices of dependence on the public health system, social vulnerability and access to health services) in a sample of individuals aged 65 and more. 688 elderly people were interviewed in a single data gathering session in their homes in 88 selected urban census sectors in Campinas. 470 of the interviewees were women, with more comorbidities and more signals and symptoms, though more socially engaged in $A A D L$ and IADL than men. Mean age was $72.28 \pm 5.41$; mean family income $=4.72 \pm 5.28$ minimum wages. The variables with most explanatory power over the joint variation of the data were access and use of health services, levels of social vulnerability and dependence on public healthcare services, and family income. Social conditions as well as family income coexist with individual vulnerability in old age.

Key words Old age, Access to health services, Socioeconomic status, Comorbidities, Functional ability
Resumo Condições socioculturais e econômicas interagem com os processos biológicos ao longo da vida, determinando vulnerabilidade ou resiliência na velhice. O objetivo foi investigar relações entre vulnerabilidade social (gênero, idade e renda); individual (comorbidades, sinais e sintomas, incapacidade funcional, suporte social percebido e saúde percebida) e programática (índices de SUSdependência e de vulnerabilidade social e acesso aos serviços de saúde) em amostra de indivíduos com 65 anos e mais. Participaram 688 idosos recrutados em domicílio, em 88 setores censitários urbanos sorteados, em Campinas, SP. Os dados (de autorrelato) foram obtidos em sessão única de coleta. Dos entrevistados, 470 eram mulheres, com mais comorbidades e sinais e sintomas, mas mais envolvidas em atividades avançadas de vida diária (AAVD) e atividades instrumentais de vida diária (AIVD) do que os homens. A média de idade foi 72,28 \pm 5,41 anos e a de renda familiar 4,72 $\pm 5,28$ salários mínimos. As variáveis que melhor explicaram a variabilidade dos dados foram acesso e uso de serviços de saúde, indices de SUS-dependência e de vulnerabilidade social e renda familiar. Condições sociais e renda familiar covariam com vulnerabilidade individual na velhice.

Palavras-chave Velhice, Acesso a serviços de saúde, Status socioeconômico, Comorbidades, Capacidade funcional 


\section{Introdução}

Vulnerabilidade é definida como o estado de indivíduos ou grupos que, por alguma razão, têm sua capacidade de autodeterminação reduzida, podendo apresentar dificuldades para proteger seus próprios interesses devido a déficits de poder, inteligência, educação, recursos, força ou outros atributos ${ }^{1,2}$. Este trabalho assume como ponto de vista norteador as três categorias de vulnerabilidade propostas por Ayres et al. ${ }^{3}$ - individual, social e programática -, para explicar a exposição de determinadas populações ao risco para doenças. A vulnerabilidade individual compreende os aspectos biológicos, emocionais, cognitivos, atitudinais e referentes às relações sociais. A social é caracterizada por aspectos culturais, sociais e econômicos que determinam as oportunidades de acesso a bens e serviços. A vulnerabilidade programática refere-se aos recursos sociais necessários para a proteção do indivíduo a riscos à integridade e ao bem-estar físico, psicológico e social. Segundo os mesmo autores, a vulnerabilidade depende da combinação dos elementos dos três domínios no momento atual. Depende também das experiências relativas a cada um deles, no passado, e de como as pessoas lidaram e lidam com as facilidades e as dificuldades da vida.

$\mathrm{O}$ envelhecimento implica em aumento do risco para o desenvolvimento de vulnerabilidades de natureza biológica, socioeconômica e psicossocial, em virtude do declínio biológico típico da senescência, o qual interage com processos socioculturais, com os efeitos acumulativos de condições deficitárias de educação, renda e saúde ao longo da vida e com as condições do estilo de vida atual. Em maior ou menor grau, aspectos individuais, coletivos, contextuais e históricos das experiências de desenvolvimento e de envelhecimento, geram possibilidades de adoecimento e dificuldades de acesso aos recursos de proteção disponíveis na sociedade.

$\mathrm{Na}$ velhice, em todos os grupos etários, há mais mulheres do que homens $s^{4-9}$. Embora mais longevas, elas são mais afetadas do que eles por doenças crônicas, incapacidade física, déficits cognitivos e emocionais ${ }^{7,10}$. Os idosos brasileiros são mais ricos do que os não-idosos ${ }^{11}$, embora a pobreza e a desigualdade social sejam condições que afetam a maioria destes últimos, principalmente os com 80 anos e mais ${ }^{12,13}$. O estudo Saúde, Bem-estar e Envelhecimento na América Latina e Caribe (SABE) identificou renda mensal média de dois salários mínimos entre os idosos brasileiros. Verificou também, que mulheres tinham um rendimento $35 \%$ inferior aos homens ${ }^{7}$.

Na velhice, a prevalência de incapacidade funcional, de déficit cognitivo, de doenças crônicas e de sintomas depressivos é maior entre os indivíduos mais velhos, as mulheres e os idosos com nível mais baixo de renda e de escolaridade ${ }^{14-18}$. O apoio social informal e a maneira como o idoso percebe a própria saúde apresentam-se como recursos protetores que facilitam a adaptação dos idosos às perdas associadas ao envelhecimento $0^{5,16,19-24}$.

Os idosos têm grande dependência dos serviços de saúde devido a doenças, incapacidades e déficits físicos e cognitivos associados ao envelhecimento. Estudos anteriores ${ }^{23,25}$ encontraram alta frequência de consultas médicas e baixa quantidade de hospitalizações no último ano em idosos que residem na comunidade. Os idosos que mais utilizam os serviços de saúde são as mulheres, os que possuem planos particulares de serviços médicos, os que têm maior número de doenças e os que têm pior autopercepção de saúde ${ }^{25}$. Em geral, os idosos mais pobres utilizam mais as clínicas e os hospitais públicos e os com melhores condições econômicas, os serviços pagos ${ }^{18,23,26}$. Os idosos com maior renda tendem a usar os serviços oferecidos pela rede privada de atendimento médico e odontológico. Há baixa oferta de serviços odontológicos públicos e conveniados. Assim, quanto mais velho e mais pobre o indivíduo, menor a frequência de visitas ao dentista e pior a qualidade da sua saúde bucal, que também se nutre dos déficits em cuidados acumulados ao longo da vida ${ }^{8,20,27}$.

Não há dados brasileiros focalizando condições com potencial para gerar vulnerabilidade social, individual e programática em idosos e nem há dados sistemáticos sobre a interação entre elas. O estudo das condições individuais, econômicas e sociais de vulnerabilidade da população idosa, em interação com o acesso e o uso dos serviços de saúde, pode auxiliar no planejamento de ações de atenção à saúde do idoso. Pode igualmente subsidiar políticas de saúde e educação para a população de crianças, jovens e adultos, uma vez que o envelhecimento é um processo de curso de vida.

A hipótese estabelecida para este trabalho é que variáveis indicadoras de vulnerabilidade programática associam-se a variáveis indicadoras de vulnerabilidade social, na determinação da vulnerabilidade individual, expressa por condições deficitárias de saúde física e psicológica em idosos comunitários urbanos com 65 anos e mais.

Os objetivos foram: a) caracterizar condições de vulnerabilidade social indicada por gênero, 
idade e renda; de vulnerabilidade individual, indicada por número de doenças, incapacidade funcional, suporte social percebido e saúde percebida, e de vulnerabilidade programática, refletida em índices de SUS-dependência e de vulnerabilidade social e em acesso e uso de serviços de saúde e b) analisar as relações conjuntas entre esses indicadores.

\section{Materiais e métodos}

Este estudo envolveu 688 idosos com 65 anos e mais, integrantes de amostra casualizada de 900 indivíduos recrutados em domicilio por equipe treinada, em 88 setores censitários urbanos sorteados da cidade de Campinas, SP, no contexto de uma investigação sobre fragilidade (Estudo FIBRA - Fragilidade em Idosos Brasileiros). Os critérios de exclusão utilizados por ocasião do recrutamento realizado em domicílio, por equipes treinadas foram: a) presença de problemas de memória, atenção, orientação espacial e temporal e comunicação, sugestivos de grave déficit cognitivo; b) incapacidade para andar, permitindo-se uso de bengala ou andador, mas não de cadeira de rodas; c) perda de força e afasia decorrentes de grave Acidente Vascular Encefálico; d) comprometimentos graves da motricidade, fala ou afetividade associados à doença de Parkinson; e) déficits de audição ou de visão graves dificultando a comunicação, e e) estar em estágio terminal ${ }^{28}$.

A coleta de dados foi conduzida por profissionais treinados, em sessão única com 40 a 120 minutos de duração, em centros comunitários, igrejas, escolas e unidades básicas de saúde. A primeira parte do protocolo continha questionários de identificação e dados sociodemográficos, o MiniExame do Estado Mental (MEEM), medidas de pressão arterial e antropométricas, coleta de sangue, exame bucal e medidas do fenótipo de fragilidade $^{29}$. As notas de corte no MEEM, conforme os critérios de Brucki et al. ${ }^{30}$ menos um desviopadrão, foram adotadas para a exclusão de idosos da segunda parte do protocolo de coleta de dados, que incluiu medidas de autorrelato sobre doenças crônicas, problemas de saúde, hábitos de vida, saúde bucal, acesso a serviços de saúde, capacidade funcional, expectativa de cuidado, sintomas depressivos, suporte social percebido, experiência de eventos estressantes, conceito de felicidade e satisfação com a vida.

O estudo foi aprovado pelo Comitê de Ética da Faculdade de Ciências Médicas da Universidade Estadual de Campinas. Todos os participan- tes assinaram um termo de consentimento livre e esclarecido.

\section{Instrumentos e medidas}

As variáveis de interesse para este trabalho foram investigadas mediante as seguintes condições.

\section{Vulnerabilidade social}

1) Idade, gênero e renda. Foram avaliadas por questões de autorrelato. As idades foram agrupadas em: $65-69 ; 70-74 ; 75-79$, e $\geq 80$. A renda foi agrupada em cinco faixas de salários mínimos $(\mathrm{SM}): \leq 1 ; 1,1$ a $3 ; 3,1$ a $5 ; 5,1$ a 10 ; e $>10$ SM.

\section{Vulnerabilidade individual}

1) Número de doenças. Nove itens dicotômicos investigaram se o idoso tinha as seguintes doenças crônicas diagnosticadas por médico, nos últimos 12 meses: doença do coração, hipertensão, AVC/isquemia/derrame; diabetes mellitus, câncer, artrite ou reumatismo, doenças dos pulmões; depressão e osteoporose.

2) Sinais e sintomas. Cinco itens dicotômicos perguntaram sobre incontinência urinária, perda de apetite, quedas, problemas de memória e distúrbios de sono.

3) Capacidade funcional indicada pelo desempenho em AAVD e em AIVD. Foi medido o envolvimento social definido pela participação em atividades avançadas de vida diária (AAVD) que colocam os idosos em contato com papeis sociais primários, permitem que sejam visto como ativos e produtivos e exigem independência e autonomia. Foram usados 13 itens estruturados a partir de Baltes et al. ${ }^{31}$; Reuben et al. ${ }^{32} \mathrm{e}$ Strawbridge et al. ${ }^{33}$ e três itens da escala de Lawton e Brody ${ }^{34}$ referentes a atividades instrumentais de vida diária (AIVD) realizadas fora do ambiente doméstico. As AAVD incluíram: (1) Fazer visitas na casa de outras pessoas, (2) Receber visitas em casa, (3) Ir à igreja para rituais religiosos ou atividades sociais ligadas à religião, (4) Participar de centro de convivência, (5) Participar de reuniões sociais, festas ou bailes, (6) Participar de eventos culturais (7) Dirigir automóvel, (8) Viagens de 1 dia (9) Viagens de duração mais longa (10) Trabalho voluntário, (11) Trabalho remunerado, (12) Participar de diretorias ou conselhos, (13) Participar de Universidade da Terceira Idade ou de outros cursos. As AIVD avaliadas fazer compras, lidar com dinheiro e usar meios de transporte perguntaram sobre o grau 
de ajuda que os idosos precisavam para desempenhá-las (nenhuma, parcial ou total). O desempenho dessas AIVD exige capacidades executivas preservadas e independência funcional ${ }^{35}$. Nas AAVD, perguntava-se se o idoso nunca havia realizado, se antes realizava e havia deixado de desempenhar, ou se ainda desempenhava cada atividade. Cada idoso teve um escore resultante da soma de respostas "ainda faço", descontadas as "nunca fiz". Nas AIVD, nenhuma ajuda foi contada como independência e necessidade de ajuda parcial ou total como dependência. A cada idoso foi atribuído um escore com variação de 0 a 16, correspondente às AAVD mantidas e ao desempenho totalmente independente das AIVD. Foram criadas três faixas para os valores indicativos das AAVD interrompidas ou das AIVD realizadas com ajuda: nenhuma, 1 ou 2 e 3 ou mais.

4) Suporte social percebido. Foi avaliado por meio de quatro itens semanticamente adaptados da ISEL (Interpersonal Support Evaluation List) ${ }^{36,37}$, referentes a suporte tangível (assistência material ou suporte instrumental); pertencimento ou suporte afetivo (identificação com alguém da rede de suporte; autoestima (comparações positivas com os outros) e suporte informativo (oferece informações, sugestões e conselhos). Foi calculado um escore com base nos tercis das amostras $(\mathrm{T} 1=16 \mathrm{e}$ T2 $=20)$. Aqueles que pontuaram $<16$ foram considerados com baixo suporte social percebido, entre 17 e 19, médio suporte social percebido e 20 pontos, alto suporte social percebido (Cronbach $\alpha=0,720$ ).

5) Saúde percebida. Foi aplicado um item de natureza escalar de cinco pontos (de 5 = "muito boa" a 1 = "muito ruim").

\section{Vulnerabilidade programática}

1) Acesso aos serviços de saúde. Foi avaliado por itens dicotômicos, escalares e de resposta estruturada sobre: tipo de serviço de saúde utilizado com maior frequência; posse de plano particular de saúde e dentário; tipo de serviço dentário utilizado; tempo da internação hospitalar mais duradoura no último ano e número de consultas médicas e de consultas ao dentista no último ano.

2) Índice Paulista de Vulnerabilidade Social/IPVS. Trata-se de indicador criado pela Secretaria Estadual de Saúde do Estado de São Pau$10^{38}$ com base em critérios socioeconômicos, entre os quais a proporção de moradores em aglomerados subnormais, de chefes de família com renda igual ou superior a 10 salários mínimos e de chefes de família com 15 anos de estudo ou mais; índices de SUS-dependência, mortalidade infantil, mães adolescentes e mortalidade por homicídios em homens de 15 a 39 anos. A Prefeitura de Campinas adaptou essa classificação para cinco grupos de vulnerabilidade: muito baixa, baixa, média, alta e muito alta.

3) Índices de SUS-dependência. São quatro níveis, de $50 \%$ a $100 \%$. Relaciona-se com a proporção de indivíduos que utilizam os serviços públicos de saúde, em comparação com a população geral.

\section{Análise de dados}

Foram desenvolvidas análises univariadas de frequência, posição e dispersão para todas as variáveis. Para comparação entre frequências por critérios de gênero, idade e renda, foram utilizados os testes qui-quadrado e Exato de Fisher. Os testes de Mann-Whitney e Kruskal-Wallis foram usados para comparar as médias ou medianas dos grupos. A relação conjunta entre as variáveis foi avaliada mediante a técnica de análise de conglomerados ${ }^{39}$, pelo método de partição. O nível de significância adotado para os testes estatísticos foi de $5 \%(p \leq 0,05)$.

\section{Resultados}

Quatrocentos e setenta participantes eram mulheres. A idade variou entre 65 e 90 anos $(\mathrm{M}=$ $72,28 \pm 5,41$ anos). A variação da renda familiar foi entre zero e $57,83 \mathrm{SM}(\mathrm{M}=4,72 \pm 5,28)$. Não foram encontradas diferenças significantes entre os percentuais de homens e mulheres nos quatro grupos etários. Houve significativamente mais idosos com mais de 80 anos nos grupos de renda mais alta e mais baixa (Tabela 1 ).

O número de doenças variou entre zero e 7 $(2,32 \pm 1,47)$ o número de problemas de saúde, de zero a $5(1,64 \pm 1,15)$. Os homens tinham média de 1,88 doenças e as mulheres, $2,53(p<0,001)$. O número de sinais e sintomas apresentou mediana de 1,0 para os homens e de 2,0 para as mulheres $(p<0,001)$. A comparação entre os grupos de idade não revelou valores significativos em relação aos indicadores de vulnerabilidade individual. Os idosos haviam abandonado ou tinham dificuldade para desempenhar em média 3,16 $\pm 2,1$ AAVD e AIVD. A média de AAVD abandonadas ou de AIVD para cujo desempenho os idosos relataram precisar de ajuda foi de 3,39 para homens e de 3,05 para mulheres $(p=0,019)$. Os 
idosos de 80 anos e mais foram os que mais perderam em termos de envolvimento social em AAVD e em desempenho de AIVD complexas ( $p$ $<0,001)$. A maioria dos idosos apresentou bons níveis de autoavaliação de saúde. Em relação ao suporte social, 251 idosos apresentaram escores altos; 270, escores médios; e 143, relataram escores baixos. Os escores em suporte social percebido não se diferenciaram estatisticamente, conforme as variáveis gênero, idade e renda (Tabela 2).

Tabela 1. Distribuição dos indicadores de vulnerabilidade social conforme a variável gênero. FIBRA Campinas, Idosos, 2008 - 2009.

\begin{tabular}{lrcr}
\hline & & \multicolumn{2}{c}{ Frequência \% } \\
\cline { 3 - 4 } \multicolumn{1}{c}{ Variáveis } & N & Mulheres Homens \\
\hline Gênero & & & \\
$\quad$ Masculino & 218 & - & - \\
Feminino & 470 & - & - \\
Grupos de idade & & & \\
65 - 69 & 250 & 37,66 & 33,49 \\
$70-74$ & 227 & 30,21 & 38,99 \\
$75-79$ & 136 & 21,49 & 16,06 \\
$\geq 80$ & 75 & 10,64 & 11,47 \\
Renda familiar em & & & \\
salários mínimos mensais & & & \\
$\leq 1,0$ & 41 & $\mathbf{8 , 1 3}$ & 4,04 \\
$1,1-3,0$ & 238 & $\mathbf{4 1 , 6 3}$ & 34,85 \\
$3,1-5,0$ & 136 & 26,60 & 27,78 \\
$5,1-10,0$ & 114 & 17,73 & $\mathbf{2 1 , 2 1}$ \\
$>10,0$ & 48 & 5,91 & $\mathbf{1 2 , 1 2 *}$ \\
\hline
\end{tabular}

${ }^{*}$ sign para $p=0,016(\chi 2=12,21 ; \mathrm{GL}=4)$
As mulheres foram ao médico com frequência significantemente maior do que os homens. O tempo de internação hospitalar variou entre 1 e 120 dias ( $6,41 \pm 13,82$ dias). O número de consultas médicas foi entre zero e $60(4,36 \pm 4,47$ consultas). O número de consultas ao dentista no último ano variou entre zero e $20(1,24 \pm 2,23)$. Houve maior presença de idosos mais velhos nos grupos de muito baixa vulnerabilidade social e com menor dependência aos serviços do SUS. Quanto maior a vulnerabilidade social de uma região, menor a renda familiar mensal e mais idosos dependiam dos serviços do SUS e não iam ao dentista. Houve um percentual maior de mulheres do que de homens em regiões de muito baixo nível de vulnerabilidade social, com menor dependência do SUS e com mais uso de planos de saúde privados. Os indicadores de vulnerabilidade programática são apresentados nas Tabelas 3 e 4.

A análise multivariada de conglomerados foi realizada para verificar a variação conjunta dos dados, com definição a priori de dois conglomerados. As variáveis que mais contribuíram para a formação desses conglomerados foram: tipo de serviço de saúde mais utilizado, que explicou $61,35 \%$ da variabilidade, ter plano privado de saúde $(47,72 \%)$, índice de SUS-dependência $(35,99 \%)$, IVS $(35,81 \%)$, renda familiar $(26,69 \%)$, e tipo de serviço dentário utilizado $(25,50 \%)$ (Tabela 5).

O percentual, estatisticamente significante, de idosos em cada conglomerado caracterizou a amostra da seguinte maneira: $36,3 \%$ dos idosos do conglomerado 1 são do sexo masculino, e $72,8 \%$ dos pertencentes ao conglomerado 2 são

Tabela 2. Distribuição dos indicadores de vulnerabilidade individual conforme gênero, idade e renda familiar. FIBRA Campinas, Idosos, 2008 - 2009.

\begin{tabular}{|c|c|c|c|c|c|c|c|c|c|c|c|c|c|}
\hline & \multirow{2}{*}{\multicolumn{3}{|c|}{ Número de doenças }} & \multirow{2}{*}{\multicolumn{3}{|c|}{ Sinais e sintomas }} & \multirow{2}{*}{\multicolumn{3}{|c|}{ Capacidade funcional }} & \multicolumn{4}{|c|}{ Saúde percebida } \\
\hline & & & & & & & & & & \multirow{2}{*}{$\begin{array}{c}\text { Muito } \\
\text { ruim/ruim }\end{array}$} & \multirow[b]{2}{*}{ Regular } & \multirow[b]{2}{*}{ Boa } & \multirow{2}{*}{$\begin{array}{l}\text { Muito } \\
\text { boa }\end{array}$} \\
\hline & $\mathbf{0}$ & $1-2$ & $\geq 3$ & $\mathbf{0}$ & $1-2$ & $\geq 3$ & 0 & $1-2$ & $\geq 3$ & & & & \\
\hline $\mathrm{N}$ & 76 & 315 & 296 & 124 & 406 & 157 & 41 & 258 & 377 & 40 & 243 & 309 & 96 \\
\hline Homens & $15,6^{\mathrm{a}}$ & $54,59^{a}$ & 29,82 & $24,77^{\text {b }}$ & $62,39^{b}$ & 12,84 & 3,3 & 35,38 & $61,32^{c}$ & 2,75 & 36,24 & 44,5 & 16,51 \\
\hline Mulheres & 8,96 & 41,79 & $49,25^{a}$ & 14,93 & 57,57 & $27,51^{b}$ & $7,33^{c}$ & $39,44^{\mathrm{c}}$ & 53,23 & 7,23 & 34,89 & 45,11 & 12,77 \\
\hline$\leq 1 \mathrm{SM}$ & 12,2 & 24,39 & 63,41 & 17,07 & 39,02 & $43,9^{d}$ & 7,5 & 32,5 & 60,0 & $7,32^{\mathrm{e}}$ & $46,34^{\mathrm{e}}$ & 41,46 & 4,88 \\
\hline $1,1-3 \mathrm{SM}$ & 9,66 & 45,8 & 44,54 & 12,18 & $61,34^{d}$ & 26,47 & 4,72 & 36,91 & 58,37 & $7,98^{\mathrm{e}}$ & 39,5 & 41,6 & 10,92 \\
\hline $3,1-5 \mathrm{SM}$ & 12,96 & 48,15 & 38,89 & 22,22 & $63,58^{d}$ & 14,2 & 7,45 & 39,13 & 53,42 & $7,36^{\mathrm{e}}$ & 35,58 & 42,33 & 14,72 \\
\hline $5,1-10 \mathrm{SM}$ & 11,4 & 44,74 & 43,86 & 21,05 & 59,65 & 19,3 & 4,42 & 36,28 & 59,29 & 3,51 & 27,19 & $51,75^{\mathrm{e}}$ & 17,54 \\
\hline$>10 \mathrm{SM}$ & 12,5 & 58,33 & 29,17 & $37,5^{d}$ & 56,25 & 6,25 & 2,22 & 46,67 & 51,11 & 0 & 18,75 & $60,42^{\mathrm{e}}$ & $20,83^{e}$ \\
\hline
\end{tabular}

${ }^{\mathrm{a}}$ sign para $p<0.001(\chi 2=24,30, \mathrm{GL}=2) ;{ }^{\mathrm{b}} \operatorname{sign}$ para $p<0.001(\chi 2=22,57, \mathrm{GL}=2) ;{ }^{\mathrm{c}} \operatorname{sign}$ para $p=0,045(\chi 2=6,22, \mathrm{GL}=2) ;{ }^{\mathrm{d}} \operatorname{sign}$ para $p<0.001$ $(\chi 2=41,18, \mathrm{GL}=8) ;{ }^{\mathrm{e}} \operatorname{sign} p=0.011($ para $2=25,90, \mathrm{GL}=12)$ 
Tabela 3. Distribuição do IVS, índice de SUS-dependência e tipo de serviço que utiliza conforme gênero, idade e renda familiar em salários mínimos. FIBRA Campinas, Idosos, 2008 - 2009.

\begin{tabular}{|c|c|c|c|c|c|c|c|c|c|c|c|c|}
\hline & $\mathrm{N}$ & Masc. & Fem. & $65-69$ & $70-74$ & $75-79$ & $\geq 80$ & $\leq 1$ & $1,1-3$ & $3,1-5$ & $5,1-10$ & $>10$ \\
\hline \multicolumn{13}{|l|}{ IVS } \\
\hline Muito baixo & 279 & 28,44 & $46,17^{a}$ & 35,2 & 36,12 & $49,26^{d}$ & $56,0^{d}$ & 36,59 & 31,09 & 33,13 & $61,4^{f}$ & $81,25^{f}$ \\
\hline Baixo & 132 & 20,64 & 18,51 & 19,6 & $22,91^{\mathrm{d}}$ & 11,03 & $21,33^{d}$ & 17,07 & 18,91 & $22,09^{f}$ & 16,67 & 14,58 \\
\hline Médio & 144 & 22,48 & 20,21 & $22,4^{d}$ & $22,91^{\mathrm{d}}$ & $21,32^{d}$ & 9,33 & 21,95 & 21,85 & $24,54^{\mathrm{f}}$ & 15,79 & 2,08 \\
\hline Alto & 59 & $13,3^{a}$ & 6,38 & 10,8 & 6,17 & 10,29 & 5,33 & 9,76 & $13,03^{\mathrm{f}}$ & 7,36 & 4,39 & 0 \\
\hline Muito alto & 74 & $15,14^{a}$ & 8,72 & $12,0^{\mathrm{d}}$ & $11,89^{d}$ & 8,09 & 8,0 & $14,63^{f}$ & $15,13^{\mathrm{f}}$ & $12,88^{\mathrm{f}}$ & 1,75 & 2,08 \\
\hline \multicolumn{13}{|c|}{ Índice de SUS dependência } \\
\hline $50 \%$ & 266 & 27,52 & $43,83^{b}$ & 33,2 & 34,36 & $47,06^{\mathrm{e}}$ & $54,67^{\mathrm{e}}$ & 31,71 & 29,41 & 31,9 & $57,89^{\mathrm{g}}$ & $81,25^{\mathrm{g}}$ \\
\hline $60 \%$ & 127 & 18,35 & 18,51 & 19,2 & $22,91^{\mathrm{e}}$ & 9,56 & 18,67 & $21,95^{\mathrm{g}}$ & 17,23 & $20,25^{\mathrm{g}}$ & 19,3 & 14,58 \\
\hline $70 \%$ & 154 & 23,39 & 21,91 & $24,0^{\mathrm{e}}$ & $24,23^{\mathrm{e}}$ & $22,06^{\mathrm{e}}$ & 12,0 & 21,95 & 23,53 & $26,99^{g}$ & 15,79 & 2,08 \\
\hline $85 \%$ & 72 & $15,6^{b}$ & 8,09 & 11,6 & 6,61 & $16,18^{\mathrm{e}}$ & 8,09 & 9,76 & $16,81^{\mathrm{g}}$ & 7,98 & 5,26 & 0 \\
\hline $100 \%$ & 69 & $15,14^{b}$ & 7,66 & $12,0^{\mathrm{e}}$ & $11,89^{\mathrm{e}}$ & 5,15 & 6,67 & $14,63^{\mathrm{g}}$ & $13,06^{\mathrm{g}}$ & $12,88^{\mathrm{g}}$ & 1,75 & 2,08 \\
\hline \multicolumn{13}{|c|}{ Tipo de serviço que utiliza } \\
\hline SUS & 314 & $58,45^{\mathrm{c}}$ & 43,18 & 49,17 & 44,44 & 46,51 & 57,97 & $70,0^{\mathrm{h}}$ & $66,22^{h}$ & 45,22 & 17,43 & 9,76 \\
\hline Plano privado & 323 & 38,16 & $54,59^{\mathrm{c}}$ & 48,33 & 52,78 & 51,94 & 37,68 & 30 & 32,89 & 52,87 & $79,82^{h}$ & $78,05^{h}$ \\
\hline Particular & 17 & 3,38 & 2,24 & 2,5 & 2,78 & 1,55 & 4,35 & 0 & 0,89 & 1,91 & 2,75 & $12,2^{h}$ \\
\hline
\end{tabular}

${ }^{\mathrm{a}}$ sign para $p<0,01(\chi 2=26,27, \mathrm{GL}=4),{ }^{\mathrm{b}} \operatorname{sign}$. Para $p<0,01(\chi 2=26,72, \mathrm{GL}=4),{ }^{\mathrm{c}}$ sign para $p<0,001(\chi 2=15,32, \mathrm{GL}=2),{ }^{\mathrm{d}}$ sign para $p=0,005$ $(\chi 2=28,32 ; \mathrm{GL}=12),{ }^{\mathrm{e}}$ sign. Para $p<0,001(\chi 2=37,11 ; \mathrm{GL}=12),{ }^{\mathrm{f}}$ sign para $p<0,001(\chi 2=80,20, \mathrm{GL}=16),{ }^{\mathrm{g}}$ sign para $p<0,001(\chi 2=87,26, \mathrm{GL}$ $=16) ;{ }^{\mathrm{h}}$ Exato de Fischer $p<0,001$

Tabela 4. Distribuição do número de consultas médicas no ano anterior, número de consultas ao dentista no último ano e tipo de serviço dentário mais utilizado conforme gênero, idade e renda familiar em salários mínimos. FIBRA Campinas, Idosos, 2008 - 2009.

\begin{tabular}{|c|c|c|c|c|c|c|c|c|c|c|c|c|}
\hline & $\mathbf{N}$ & Masc. & Fem. & 65-69 & $70-74$ & $75-79$ & $\geq 80$ & $\leq 1$ & $1,1-3$ & $3,1-5$ & $5,1-10$ & $>10$ \\
\hline \multicolumn{13}{|c|}{$\begin{array}{l}\text { Número de consultas } \\
\text { médicas no último ano }\end{array}$} \\
\hline 0 & 37 & $10,0^{a}$ & 3,84 & $8,09^{b}$ & 4,65 & 2,29 & $8,06^{\mathrm{b}}$ & 2,78 & 5,75 & 5,84 & 2,68 & 16,67 \\
\hline $1-2$ & 198 & $33,0^{a}$ & 29,8 & 33,62 & 23,72 & $37,4^{b}$ & 30,65 & 30,56 & 32,3 & 32,47 & 31,25 & 19,05 \\
\hline$\geq 3$ & 408 & 57 & $66,37^{a}$ & 58,3 & $71,63^{b}$ & 60,31 & 61,29 & 66,67 & 61,95 & 61,69 & 66,07 & 64,29 \\
\hline \multicolumn{13}{|c|}{$\begin{array}{l}\text { Número de consultas ao } \\
\text { dentista no último ano }\end{array}$} \\
\hline 0 & 338 & 53,33 & 48,81 & 49,59 & 50,67 & 47,01 & 57,14 & $55,0^{c}$ & $64,22^{\mathrm{c}}$ & 44,72 & 36,84 & 22,73 \\
\hline $1-2$ & 240 & 31,43 & 37,58 & 35,66 & 32,44 & 41,79 & 34,29 & 37,5 & 28,88 & 37,27 & $49,1^{\mathrm{c}}$ & 36,36 \\
\hline$\geq 3$ & 95 & 15,24 & 13,61 & 14,75 & 16,89 & 11,19 & 8,57 & 7,5 & 6,9 & 18,01 & 14,04 & $40,91^{\mathrm{c}}$ \\
\hline \multicolumn{13}{|c|}{ Tipo de serviço dentário } \\
\hline SUS & 210 & 37,44 & 30,25 & 36,86 & 29,95 & 28,35 & 33,33 & $56,41^{d}$ & $45,87^{d}$ & 28,93 & 12,73 & 4,65 \\
\hline Plano privado & 55 & 8,87 & 8,35 & 7,2 & 11,98 & 6,3 & 6,06 & 2,56 & 7,8 & 8,81 & 8,18 & $20,93^{d}$ \\
\hline Particular & 381 & 53,69 & 61,4 & 55,93 & 58,06 & 65,35 & 60,61 & 41,03 & 46,33 & $62,26^{d}$ & $79,09^{d}$ & $74,42^{d}$ \\
\hline
\end{tabular}

${ }^{\mathrm{a}} \operatorname{sign}$ para $p=0,003(\chi 2=11,46, \mathrm{GL}=2) ;{ }^{\mathrm{b}} \operatorname{sign}$ para $p=0,017(\chi 2=15,44 ; \mathrm{GL}=6),{ }^{\mathrm{c}} \operatorname{Sign}$ para $p<0,001(\chi 2=64,24, \mathrm{GL}=8),{ }^{\mathrm{d}} \operatorname{Sig}$ para $p<0,001$ $(\chi 2=70,05, \mathrm{GL}=8)$.

do sexo feminino $\left(p=0,011 ; \chi^{2}=6,52 ; G L=1\right)$. $87,4 \%$ dos idosos do grupo 1 usam os serviços do SUS, ante $91 \%$ do grupo 2, que usam plano privado e serviços particulares $\left(p<0,001 ; \chi^{2}=\right.$ 401,51; GL = 2); no conglomerado $1,70,5 \%$ dos idosos vivem em regiões com altos níveis de SUSdependência e no conglomerado 2, 64,76\% mo- ram em áreas com índices mais baixo de SUSdependência $\left(p<0,001 ; \chi^{2}=267,74 ; \mathrm{GL}=4\right)$; $66,18 \%$ dos idosos do conglomerado 1 vivem em regiões com nível mais alto de vulnerabilidade social e $67,34 \%$ daqueles do conglomerado 2 vivem em regiões com nível mais baixo de vulnerabilidade social $\left(p<0,001 ; \chi^{2}=261,25 ; \mathrm{GL}=4\right)$; 
Tabela 5. Resultados da análise de conglomerados $(\mathrm{n}=688)$. FIBRA Campinas, Idosos, 2008 - 2009.

\begin{tabular}{lcccc}
\hline \multicolumn{1}{c}{ Variável } & DP total & Desvio padrão & Coeficiente de determinação $\left(\mathbf{R}^{2)}\right.$ & RSQ/(1-RSQ) \\
\hline Gênero & 1,000 & 0,99597 & 0,009484 & 0,009574 \\
Idade & 1,000 & 0,99954 & 0,002382 & 0,002387 \\
Renda & 1,000 & 0,85692 & $\mathbf{0 , 2 6 6 9 1 0}$ & 0,364088 \\
IVS & 1,000 & 0,80176 & $\mathbf{0 , 3 5 8 1 1 4}$ & 0,557909 \\
Internação hospitalar & 1,000 & 0,99833 & 0,004824 & 0,004847 \\
Consultas médicas & 1,000 & 0,99119 & 0,019067 & 0,019437 \\
Serviço de saúde & 1,000 & 0,62216 & $\mathbf{0 , 6 1 3 5 0 5}$ & 1,587353 \\
Plano de saúde & 1,000 & 0,72355 & $\mathbf{0 , 4 7 7 2 5 8}$ & 0,912990 \\
Consulta ao dentista & 1,000 & 0,96467 & 0,070799 & 0,076194 \\
Serviço dentário & 1,000 & 0,86379 & $\mathbf{0 , 2 5 5 0 3 1}$ & 0,342337 \\
SUS-dependência & 1,000 & 0,80058 & $\mathbf{0 , 3 5 9 9 9 9}$ & 0,562498 \\
Doenças & 1,000 & 0,99890 & 0,003657 & 0,003671 \\
Problemas de saúde & 1,000 & 0,98055 & 0,039921 & 0,041581 \\
Capacidade funcional & 1,000 & 0,99957 & 0,002350 & 0,002355 \\
Suporte social & 1,000 & 0,99366 & 0,014123 & 0,014326 \\
Saúde percebida & 1,000 & 0,97729 & 0,046295 & 0,048542 \\
Total & 1,000 & 0,91862 & $\mathbf{0 , 1 5 7 3 9 4}$ & 0,186794 \\
\hline
\end{tabular}

69,35\% dos idosos do conglomerado 1 têm renda familiar até 3 salários mínimos e 67,34\% dos do grupo 2 têm renda maior que 5,1 salários mínimos $\left(p<0,001 ; \chi^{2}=169,81 ; \mathrm{GL}=4\right) ; 58,15 \%$ dos idosos do grupo 1 usam serviços dentários do SUS ante $91,59 \%$ dos idosos do grupo 2, que usam serviços particulares ou de convênios odontológicos $\left(p<0,001 ; \chi^{2}=181,95 ; \mathrm{GL}=2\right)$. No conglomerado $1,43,8 \%$ dos entrevistados foram a até 2 consultas médicas no ano anterior e 70,3\% dos idosos do grupo 2 consultaram médico 3 ou mais vezes no ano anterior à entrevista $(p=0,001$; $\chi^{2}=13,74 ; \mathrm{GL}=2$ ). Ao mesmo tempo, no grupo $1,61,7 \%$ dos idosos não consultaram o dentista, no grupo 2, 39,3\% realizaram 1 ou 2 consultas e $21,7 \%$ dos idosos foram 3 ou mais vezes ao dentista no ano anterior $\left(p<0,001 ; \chi^{2}=48,06\right.$; GL $=$ 2). Dos idosos pertencentes ao grupo $1,30,2 \%$ relatam 3 ou mais sinais e sintomas, enquanto $23,5 \%$ do grupo 2 apresentam nenhum sinal ou sintoma $\left(p<0,001 ; \chi^{2}=27,6, \mathrm{GL}=2\right) ; 25,9 \%$ no conglomerado 2 apresentam baixo suporte social, enquanto $42,3 \%$ no conglomerado 2 têm alto suporte social percebido $\left(p=0,009 ; \chi^{2}=9,5, \mathrm{GL}\right.$ $=2)$; no grupo $1,51 \%$ dos entrevistados percebem de muito ruim a regular saúde, enquanto $68,5 \%$ do grupo 2 teve boa ou muito boa saúde percebida $\left(\mathrm{p}<0,001 ; \chi^{2}=33,67 ; \mathrm{GL}=4\right)$.

Em comparação com o conglomerado 2, o 1 foi caracterizado por maior proporção de idosos do gênero masculino e de idosos com menor renda familiar, residentes em regiões de maior vulnerabilidade social, que relataram ter comparecido a um menor número de consultas médicas, que são assistidos predominantemente pelo SUS, que não têm plano particular de saúde, que foram a um menor número de consultas odontológicas no último ano, que usam mais serviços dentários do SUS, que moram em áreas com maior dependência ao SUS, que relataram ter maior número de problemas de saúde, que avaliaram de forma mais negativa o suporte social de que dispõem e que avaliaram a própria saúde como ruim ou muito ruim.

Em comparação com o conglomerado 1, o 2 foi composto por um maior percentual de mulheres idosas, assim como por maior percentual de idosos com maior renda familiar, com residência em áreas de menor vulnerabilidade social, com maior número de consultas médicas no ano anterior, com acesso a planos privados de saúde ou a serviços particulares, com maior número de consultas odontológicas no ano anterior, que utilizam mais serviços dentários particulares ou que têm planos privados de assistência odontológica, que moram em regiões com menor dependência ao SUS-dependência, que têm menor número de problemas de saúde e que avaliam mais positivamente o suporte social e a própria saúde.

\section{Discussão}

A qualidade de vida na velhice depende das experiências individuais e coletivas ao longo da vida. Indivíduos expostos a carências graves durante a infância e a adolescência têm seu desenvolvimento 
prejudicado por danos acumulativos que se refletem nas suas condições físicas, nas atitudes e nos demais elementos pertencentes ao domínio de vulnerabilidade que, neste trabalho, foi considerado como o individual, assim como se refletem no acesso e no uso de serviços sociais e de saúde ${ }^{13,40,41}$.

Foi encontrado maior número de mulheres do que de homens, de forma semelhante a outras pesquisas ${ }^{4,7-9,16}$, refletindo o fenômeno de feminização da velhice ${ }^{10}$. A frequência de mulheres superou a de outros estudos, talvez pela forma de recrutamento, realizado principalmente por agentes comunitárias, em sua maioria mulheres. Outra possibilidade de explicação para esse dado é que geralmente as mulheres têm maiores adesão a tratamentos e a informações sobre saúde.

Os idosos com 80 anos ou mais representaram $10,9 \%$ da amostra, proporção inferior à observada na população de Campinas ${ }^{6}$, talvez devido aos critérios de exclusão, talvez por sua maior dificuldade em comparecer aos locais de coleta de dados. Segundo Barros ${ }^{11}$, há relação positiva entre renda e expectativa de vida, explicando a maior presença de idosos mais velhos no grupo com renda mais alta observada neste estudo. Provavelmente os octogenários que compareceram à sessão de coleta de dados teriam melhores condições de saúde ou teriam desenvolvido suficientes mecanismos de enfrentamento ao longo da vida, que lhes permitiram transformar-se em sobreviventes relativamente bem-sucedidos ${ }^{13}$.

Confirmando dados da literatura ${ }^{7,10,42}$, as mulheres tinham menor renda do que os homens, possivelmente devido às desvantagens educacionais e empregatícias que fizeram com que, na velhice, a maioria delas não tivesse renda. Essa ocorrência é ainda muito comum, mesmo a renda das idosas brasileiras tendo aumentado nos últimos anos, por causa da universalização das aposentadorias e pensões e por causa da oferta do beneficio de prestação continuada aos idosos muito pobres.

A frequência de comorbidades ou a ausência de doenças na amostra foi comparável a de outros estudos brasileiros ${ }^{16,18}$, assim como a maior presença de doenças e de sinais e sintomas entre as mulheres ${ }^{14,16}$. Os idosos com menor renda apresentaram mais sinais e sintomas, provavelmente devido à falta de acesso e de atenção à saúde durante a vida. A interrupção de AAVD ou a necessidade de ajuda para as três AIVD avaliadas foi mais comum entre os idosos mais velhos, concordando com outras pesquisas ${ }^{14,16,24}$. Porém, diferentemente da literatura ${ }^{15,17}$, houve mais homens do que mulheres que interromperam ou passaram a necessitar de ajuda para essas ativi- dades. Provavelmente os homens idosos têm menor interesse e participação em grupos sociais e em atividades sociais realizadas fora de casa ${ }^{43}$. Foram utilizadas questões referentes às AAVD e AIVD, que, segundo Fonseca e Rizzotto ${ }^{44}$, são atividades mais complexas e relacionadas ao envolvimento social e que não necessariamente são indicadores de competência física. Neri et al. ${ }^{45}$ observaram que homens idosos que cuidavam de outros idosos afastaram-se de mais AAVD do que mulheres cuidadoras e avaliaram o suporte social de forma mais negativa do que elas. Essa ocorrência chama a atenção para um aspecto pouco explorado das vivências dos idosos na relação com o envolvimento social e com a preservação da capacidade funcional na realização de atividades complexas de vida diária. Para os idosos, a qualidade de vida tem menos relação com a presença de comorbidades do que com a funcionalidade e com o modo como se adaptam às perdas físicas e cognitivas decorrentes do envelhecimento. A independência funcional está associada com boa saúde, participação social e qualidade de vida na velhice ${ }^{17,41}$.

As avaliações altamente positivas do suporte social pela grande maioria dos idosos são semelhantes ao estudo de Guedea et al. ${ }^{5}$. Subjacentes a essas avaliações pode estar o fenômeno de intercâmbio de ajuda entre gerações, cada vez mais comum nas famílias brasileiras com idosos ${ }^{13}$. Por outro lado, a convivência intergeracional em famílias economicamente muito vulneráveis pode expor o idoso a prejuízos no acesso a recursos para custeio de remédios e de outros tratamentos de saúde.

As autoavaliações de saúde foram altamente positivas, um dado que concorda com os da literatura ${ }^{16,20,21,23}$. Houve mais idosos com melhor autopercepção de saúde entre aqueles com renda mais alta e mais idosos com pior autopercepção de saúde entre os com renda mais baixa, resultados também encontrados em outras pesquisas $^{16,21,24}$. A boa autopercepção de saúde, mesmo na presença de indicadores de vulnerabilidade, pode ser explicada por mecanismos de autorregulação cognitivo-emocionais, que podem favorecer a adesão a tratamentos de saúde e a hábitos de saúde, entre eles a prática de exercícios físicos e alimentação equilibrada ${ }^{24,46,47}$.

A baixa frequência de idosos vivendo em regiões de alta vulnerabilidade social e baixa dependência aos serviços do SUS estão relacionados ao alto IDH de Campinas, que, em comparação com outras cidades do Estado de São Paulo, tem maior percentual de sua população residente em regi- 
ões de muito baixa e nenhuma vulnerabilidade social $^{38}$. Ainda assim, a desigualdade social é muito presente, chamando a atenção para a necessidade de políticas adequadas para minimizar essa condição. Dados internacionais mostram que a desigualdade social é mais determinante de vulnerabilidades em saúde física e psicológica do que níveis absolutos de pobreza ${ }^{48,49}$.

A quantidade de internações hospitalares foi baixa, à semelhança dos dados de Lima-Costa e Loyola Filho ${ }^{25}$. Esse resultado talvez reflita bons níveis de saúde associados à renda relativamente alta de parte dos idosos, mas nos grupos sociais mais vulneráveis, pode indicar deficiências no acesso a serviços de diagnóstico e tratamento de saúde. $\mathrm{Na}$ amostra, 63,45\% submeteram-se a três ou mais consultas médicas no último ano, concordando com a literatura ${ }^{15,23}$. A frequência elevada destas pode ser positiva, caso tenham sido realizadas como forma de prevenção e manutenção da saúde. Dependendo da demora para a marcação das consultas e da qualidade dos atendimentos, acompanhamentos e encaminhamentos, o maior número de consultas pode ser desgastante para os idosos e pode significar acesso inadequado aos serviços.

Segundo Lima-Costa ${ }^{50}$, os idosos mais velhos e com renda mais baixa têm pior acesso aos serviços de saúde. Nesta pesquisa, houve frequência mais baixa de uso de serviços de saúde pelos idosos de 65 a 69 anos e por aqueles com 80 anos ou mais. Os mais jovens teriam ido pouco ao médico por terem melhor saúde, enquanto os mais velhos teriam ido menos por causa da pobreza e da falta de ajuda instrumental. Outros estudos mostraram relações entre piores condições econômicas e maior dependência dos serviços públicos de saúde ${ }^{18,23,25,26}$.

Metade dos entrevistados utilizava serviço dentário particular, semelhante ao estudo de Benedetti ${ }^{20}$, mas como uma proporção comparável não havia ido ao dentista no ano anterior, conclui-se que há um descompasso entre as necessidades da população e os serviços prestados. Esta impressão é reforçada pela evidência de que os idosos mais pobres e os que viviam em regiões de maior IVS foram menos ao dentista e utilizaram mais os serviços do SUS, enquanto os mais ricos utilizaram mais os serviços particulares, como notado por outros estudos ${ }^{8,27}$.

As variáveis que mais explicaram a variação conjunta dos dados pertencem ao domínio da vulnerabilidade programática. A renda associouse com elas, como era de se esperar. Essas relações confirmam o raciocínio expresso na hipótese norteadora deste estudo.

\section{Conclusões}

A análise de dados foi de natureza descritiva e não inferencial, não permitindo tirar conclusões sobre o impacto relativo dos três conjuntos de condições de vulnerabilidade e nem tampouco de variáveis isoladas sobre uma ou mais variáveis dependentes. No entanto, mesmo sendo de natureza explo-ratória, os dados do presente trabalho permitiram chegar a informações úteis, que poderão ser testados em futuras análises.

$\mathrm{O}$ estudo teve limitações referentes à forma de recrutamento, feito majoritariamente por agentes comunitárias, o que terá contribuído para privilegiar a participação feminina. Os critérios de exclusão adotados terão favorecido a participação de idosos mais saudáveis, ativos e envolvidos socialmente.

Sugere-se que sejam realizados novos estudos de corte transversal envolvendo as relações entre as variáveis dentro de cada domínio de vulnerabilidade, assim como estudos longitudinais, para entender a relação entre os diferentes domínios da vulnerabilidade com o passar dos anos. Estudos de sequencia de coortes permitirão entender a influência de variáveis sócio-históricas.

A divulgação destes dados a gestores de serviços de saúde, tanto em unidades básicas como em polos regionais, poderá subsidiar decisões sobre prioridades, aplicação de recursos e treinamento de pessoal na atenção ao idoso.

\section{Colaboradores}

$\mathrm{N}$ de $\mathrm{O}$ Rodrigues trabalhou na concepção e na redação do artigo, sob a orientação de AL Neri, que coordenou o Estudo Fibra Unicamp e o Estudo Fibra Campinas, planejou a análise de dados e revisou do texto.

\section{Agradecimentos}

A Mônica Sanches Yassuda pela colaboração no planejamento do Estudo FIBRA polo Unicamp, juntamente com Andréa Cristina Garofe FortesBurgos e Efigênia Passarelli Mantovani, que também atuaram na coordenação da coleta de dados em Campinas, ao lado de Flávia Silva Arbex, Stella Vital de Souza Torres e Débora dias da Silva Harmitt. 


\section{Referências}

1. Barchifontaine CP. Vulnerabilidade e dignidade humana. O Mundo da Saúde 2006; 30(3):434-440.

2. Neves MCP. Sentidos da vulnerabilidade: característica, condição, princípio. In: Barchifontaine CP, Zoboli ELCP, organizadores. Bioética, vulnerabilidade e saúde. São Paulo: Editora Centro Universitário São Camilo; 2007. p. 29-45.

3. Ayres J, Calazans GJ, Saletti Filho HC, França Jr I. Risco, vulnerabilidade e práticas de prevenção e promoção da saúde. In: Campos G, Minayo MCS, Akerman M, Drumond Jr M, Carvalho YM, organizadores. Tratado de Saúde Coletiva. São Paulo: Editora Fiocruz; 2006. p. 375-417.

4. Fung HH, Yeung DY, Li KK, Lang FR. Benefits of negative social exchanges for emotional closeness. J Gerontol B Psychol Sci Soc Sci 2009; 64(5):612-621.

5. Guedea M, Albuquerque FJB, Tróccoli BT, Noriega JAV, Seabra MAB, Guedea RLD. Relação do bemestar subjetivo, estratégias de enfrentamento e apoio social em idosos. Psicologia, Reflexao e Critica 2006; 19(2):301-308.

6. Brasil. Instituto Brasileiro de Geografia e Estatística. Síntese de Indicadores Sociais: Uma Análise das Condições de Vida da População Brasileira, 2008. [site na Internet]. [acessado 2010 jul 05]. Disponível em: http://www.ibge.gov.br/

7. Lebrão M, Duarte, YAO, organizadoras. SABE - Saúde, Bem-estar e Envelhecimento. O Projeto Sabe no Município de São Paulo: uma abordagem inicial. Brasília: Organização Pan-Americana de Saúde; 2003.

8. Matos D, Lima-Costa, MF. Tendência na utilização de serviços odontológicos entre idosos brasileiros e fatores associados: um estudo baseado na Pesquisa Nacional por Amostra de Domicílios (1998 e 2003). Cad Saude Publica 2007; 23(11):2740-2748.

9. Mazzella F, Cacciatore F, Galizia G, Della-Morte D, Rossetti M, Abbruzzese R, Langellotto A, Avolio D, Gargiulo G. Ferrara N, Rengo F, Abete, P. Social support and long-term mortality in the elderly: role of comorbidity. Arch Gerontol Geriatr 2010; 51(3): 323-328.

10. Neri A. Feminização da velhice. In: Abramo FP, editor. Idosos no Brasil: Vivências, desafios e expectativas na terceira idade. São Paulo: Editora SESCSP; 2007. p. 47-64.

11. Barros R, Mendonça R, Santos D. Incidência e natureza da pobreza entre idosos no Brasil. In: Camarano A, organizadora. Muito Alem dos 60 - Os Novos Idosos Brasileiros. Rio de Janeiro: Editora IPEA; 1999. p. 221-249.

12. Marmot M, Friel S, Bell R, Houweling TA, Taylor S. Closing the gap in a generation: health equity through action on the social determinants of health. Lancet 2008; 372(9650):1661-1669.

13. Camarano A, Kanso S, Mello JL. Como vive o idoso brasileiro? In: Camarano A, organizadora. Os novos idosos brasileiros: Muito além dos 60? Rio de Janeiro: Editora IPEA; 2004. p. 25-73.
14. Barros M, César CLG, Carandina L, Torre GD. Desigualdades sociais na prevalência de doenças crônicas no Brasil, PNAD-2003. Cien Saude Colet 2006; 11(4):911-926.

15. Fiedler M, Peres, KG. Capacidade funcional e fatores associados em idosos do Sul do Brasil: um estudo de base populacional. Cad Saude Publica 2008; 24(2):409-415.

16. Brasil. Instituto Brasileiro de Geografia e Estatística. IBGE. Síntese de Indicadores Sociais: Uma Análise das Condições de Vida da População Brasileira, 2010. [site na Internet] [acessado 2011 maio 12]. Disponível em: http://www.ibge.gov.br/

17. Santos K, Koszuoski R, Dias-da-Costa JS, Pattussi MP. Fatores associados com a incapacidade funcional em idosos do Município de Guatambu, Santa Catarina, Brasil. Cad Saude Publica 2007; 23(11):27812788.

18. Veras R, Parahyba M I. Anacronismo dos modelos assistenciais para os idosos na área da saúde. Cad Saude Publica 2007; 23(10):2479-2489.

19. Berg C, Smith TW, Henry NJM, Pearce GE. A developmental approach to psychological risk factors and successful aging. In: Aldwin C, Park CL, Spiro A, editors. Handbook of Health Psychology and Aging. New York: Guilford Press; 2007. p. 30-53.

20. Benedetti T, Mello ALSF, Gonçalves LHT. Idosos de Florianópolis: autopercepção das condições de saúde bucal e utilização de serviços odontológicos. Cien Saude Colet 2007; 12(6):1683-1690.

21. Chittleborough CR, Taylor AW, Baum FE, Hiller JE. Monitoring inequities in self-rated health over the life course in population surveillance systems. Am J Public Health 2009; 99(4):680-689.

22. De Vitta A, Neri AL, Padovani CR. Saúde percebida em homens e mulheres sedentários e ativos, adultos jovens e idosos. Salusvita 2006; 25(1):23-34.

23. Louvison M, Lebrão ML, Duarte YAO, Santos JLF, Malik AM, Almeida ES. Desigualdades no uso e acesso aos serviços de saúde entre idosos do município de São Paulo. Rev Saude Publica 2008; 42(4): 733-740.

24. Martin LG, Schoeni RF, Freedman VA, Andreski P. Feeling better? Trends in general health status. $J$ Gerontol B Psychol Sci Soc Sci 2007; 62(1):S11-21.

25. Lima-Costa M, Loyola-Filho AI. Fatores associados ao uso e à satisfação com os serviços de saúde entre usuários do Sistema Único de Saúde na Região Metropolitana de Belo Horizonte, Estado de Minas Gerais, Brasil. Epidemiologia e Serviços de Saúde 2008; 17(4):247-258.

26. Pessoto U, Kayano J, Ibanhes LC, Junqueira V, Barboza R, Cortizo CT. Desigualdades no acesso e utilização dos serviços de saúde na Região Metropolitana de São Paulo. Cien Saude Colet 2007; 12(2): 351-362. 
27. Araújo C, Lima RC, Peres MA, Barros AJD. Utilização de serviços odontológicos e fatores associados: um estudo de base populacional no Sul do Brasil. Cad Saude Publica 2009; 25(5):1063-1072.

28. Ferrucci L, Guralnik JM, Studenski S, Fried LP, Cutler Junior GB, Walston JD. Designing randomized, controlled trials aimed at preventing or delaying functional decline and disability in frail, older persons: a consensus report. J Am Geriatr Soc 2004; 52(4):625-634.

29. Fried LP, Tangen CM, Walston J, Newman AB, Hirsch C, Gottdiener J, Seeman T, Russell T, Kop WJ, Burke G, McBurnie MA. Frailty in older adults: evidence for a phenotype. J Gerontol A Biol Sci Med Sci 2001; 56(3):M146-156.

30. Brucki SMD, Nitrini R, Caramelli P, Bertolucci PHF, Okamoto IH. Sugestões para o uso do Mini-Exame do Estado Mental no Brasil. Arq Neuropsiquiatr 2003; 61(3-B):777-781.

31. Baltes MM, Mayr U, Borchelt M, Maas I, Wilms HU. Everyday competence in old and very old age: An interdisciplinary perspective. Ageing Soc 1993; 13(4):657-680.

32. Reuben DB, Laliberte L, Hiris J, Mor V. A hierarchical exercise scale to measure function at the Advanced Activities of Daily Living (AADL) level. J Am Geriatr Soc 1990; 38(8):855-861.

33. Strawbridge WJ, Wallhagen MI, Cohen RD. Successful aging and well-being: self-rated compared with Rowe and Kahn. Gerontologist 2002; 42(6):727-733.

34. Lawton MP, Brody EM. Assessment of older people: self-maintaining and instrumental activities of daily living. Gerontologist 1969; 9(3):179-186.

35. Pereira FS, Yassuda MS, Oliveira AM, Diniz BS, Radanovic M, Talib LL, Gattaz WF, Forlenza OV. Profiles of functional deficits in mild cognitive impairment and dementia: benefits from objective measurement. J Int Neuropsychol Soc 2010; 16(2):297305.

36. Cohen S, Mermelstein R, Kamarck T, Hoberman HM. Measuring the functional components of social support. In: Sarason G, Sarason BR, editors. Social support: Theory, research, and applications. The Hague: Martinus Nijhoff; 1985. p. 73-94.

37. Batistoni SST, Neri AL, Cupertino APFB. Validade da escala de depressão do Center for Epidemiological Studies (CES-D) entre idosos brasileiros. Rev Saude Publica 2007; 41(4):598-605.

38. Fundação Sistema Estadual de Análise de Dados. Índice Paulista de Vulnerabilidade Social/IPVS. São Paulo, 2000. [site na Internet]. [acessado 2010 jun 16]. Disponível em: http://www.seade.gov.br/produtos/ipvs/apresentacao.php

39. Pereira JCR. Análise de Dados Qualitativos - Estratégias Metodológicas para as Ciências da Saúde, Humanas e Sociais. São Paulo: Editora EDUSP; 1999.
40. Engler T. Como a economia pode favorecer uma velhice bem sucedida. In: Neri AL, organizadora. Qualidade de vida na velhice: enfoque multidisciplinar. São Paulo: Editora Alínea; 2007. p. 83-126.

41. Fortes-Burgos ACGF. Condições de risco biológico e psicossocial, recursos psicológicos e sociais e funcionalidade em idosos residentes na comunidade [tese]. Campinas (SP): Universidade Estadual de Campinas; 2010.

42. Silva MC. O processo de envelhecimento no Brasil: desafios e perspectivas. Textos Envelhecimento 2005; 8(1):43-60.

43. Areosa SVC. O que pensam as mulheres e os homens idosos sobre o seu envelhecimento? Textos e Contextos 2004; 3:1-10.

44. Fonseca FB, Rizzotto MLF. Construção de instrumento para avaliação sócio-funcional em idosos. Texto e Contexto Enfermagem 2008; 17(2):365-373.

45. Neri AL, Yassuda MS, Garofe-Fortes AC, Mantovani EP, Arbex FS, Torres SVS, Perracini MR, Guariento ME. Relationships between gender, age, family conditions, physical and mental health, and social isolation of elderly caregivers. Int Psychogeriatr 2011; 24(3): 472-483.

46. Blazer DG. How do you feel about...? Health outcomes in late life and self-perceptions of health and well-being. Gerontologist 2008; 48(4):415-422.

47. Liang J, Shaw BA, Krause N, Bennett JM, Kobayashi E, Fukaya T, Sugihara Y. How does self-assessed health change with age? A study of older adults in Japan. J Gerontol B Psychol Sci Soc Sci 2005; 60(4):S224-232.

48. Ladin K, Daniels N, Kawachi I. Exploring the relationship between absolute and relative position and late-life depression: evidence from 10 European countries. Gerontologist 2009; 50(1):48-59.

49. Lorant V, Deliege D, Eaton W, Robert A, Philippot P, Ansseau M. Socioeconomic inequalities in depression: a meta-analysis. Am J Epidemiol 2003; 157(2):98-112.

50. Lima-Costa MF. Influência da idade e escolaridade no uso de serviços preventivos de saúde. - Inquérito de Saúde da Região Metropolitana de Belo Horizonte, Minas Gerais, Brasil. Epidemiologia e serviços de saúde 2004; 13(4):209-215.

Artigo apresentado em 31/08/2011

Aprovado em 15/09/2011

Versão final apresentada em 29/09/2011 